\title{
Assessment of explanatory models of mental illness: effects of patient and interviewer characteristics
}

\author{
Samrad Ghane $\cdot$ Annemarie M. Kolk • \\ Paul M. G. Emmelkamp
}

Received: 12 January 2009/Accepted: 31 March 2009/Published online: 21 April 2009

(c) The Author(s) 2009. This article is published with open access at Springerlink.com

\begin{abstract}
Background Explanatory models (EMs) refer to patients' causal attributions of illness and have been shown to affect treatment preference and outcome. Reliable and valid assessment of EMs may be hindered by interviewer and respondent disparities on certain demographic characteristics, such as ethnicity. The present study examined (a) whether ethnic minority patients reported different EMs to ethnically similar interviewers in comparison with those with a different ethnicity, and (b) whether this effect was related to respondents' social desirability, the perceived rapport with the interviewer and level of uncertainty toward their EMs.

Methods A total of 55 patients of Turkish and Moroccan origins with mood and anxiety disorders were randomly assigned to ethnically similar or dissimilar interviewers. EMs were assessed, using a semi-structured interview, across 11 different categories of causes.

Results Participants who were interviewed by an ethnically similar interviewer perceived interpersonal, victimization and religious/mystical causes as more important, whereas interviews by ethnically dissimilar interviewers generated higher scores on medical causes. These effects were not mediated by the perceived rapport with the interviewer, and social desirability had a modest impact on the results. Higher uncertainty among participants toward medical and religious/mystical causes seemed to be associated with greater adjustment in the report of these EMs. Conclusion The findings have significant implications for interviewer selection in epidemiological research and clinical practice.
\end{abstract}

S. Ghane $(\bowtie)$ - A. M. Kolk · P. M. G. Emmelkamp

Department of Clinical Psychology, University of Amsterdam,

Roetersstraat 15, 1018 WB Amsterdam, The Netherlands

e-mail:s.ghane@uva.nl
Keywords Explanatory models - Mental illness . Interviewer effect · Match effect · Ethnic minorities

\section{Introduction}

Explanatory models (EMs) refer to causal attributions of a specific episode of illness that are held by patients, their family or practitioners. Predominantly culturally shaped, these models project personal and social meaning on the illness experience [20], and can affect coping [10, 29], treatment preferences [30], compliance [16], therapeutic relationship [23] and treatment satisfaction [8]. In light of the available evidence, fostering the effectiveness of mental health care requires an understanding of patients' perspective through methodologically rigorous assessments of their EMs [5]. The present study aimed to investigate the effect of patient and interviewer characteristics on the assessment of EMs among Dutch mental health patients of Turkish and Moroccan origins.

A major obstacle in the assessment of EMs is the respondents' occasional tendency to conceal or misreport their beliefs in an interview situation. Indeed, it has been suggested that lay persons may not volunteer their EMs to clinicians, as these ideas may seem mistaken or even primitive from a Western medical point of view [20], or may simply adjust their accounts in order to appear more intelligible [34]. Thus far, little is known about the nature and magnitude of such misrepresentations, and specific factors, contributing to their occurrence, remain yet to be discovered.

In social psychological literature, misreports of beliefs and attitudes have often been ascribed to self-presentation demand [7], the tendency of respondents to present themselves in a socially desirable manner. Recently, an 
alternative interpretation has been proposed, the so-called social tuning hypothesis [31]. This hypothesis postulates that, in an attempt to form or maintain a desirable bond, individuals may adjust their beliefs and utterances in order to create a closer match with the presumed attitudes and beliefs of the interviewer. Although such tunings of one's accounts may often be driven by strategic self-presentation motives, evidence suggests that these adjustments could also, in part, be unintended [22]. Literature proposes a number of factors, which are thought to impact the adjustment and tuning of respondents' accounts. Firstly, social tuning is more pronounced among individuals with high tendency toward social desirable responding [24]. Secondly, the quality of rapport between the respondent and the interviewer is widely considered instrumental in reducing the former's self-presentation tendencies [9]. Thirdly, adjustment of attitudes and belief utterances seem predominately to occur in sensitive topics (e.g., racial prejudice) [36]. That is to say, reports are more prone to adjustment, insofar as they are perceived to provoke social rejection in the interview context. Fourthly, the adjustment of belief utterances appears to be related to respondents' level of uncertainty toward those beliefs; tuning is stronger among those who experience greater ambivalence toward their beliefs [15]. Finally, adjustments of reports may occur as a result of disparities between respondent and interviewer characteristics in terms of age, gender, status and ethnicity.

Focusing on the latter variable, one can expect ethnic minority patients to produce different accounts with ethnically similar (ethnic match) and dissimilar interviewers (ethnic mismatch). Such ethnic match effects have been widely demonstrated in studies into racial and political attitudes (e.g., [2, 12]), and only marginally tested in mental health research. Thompson, Worthington, and Atkinson [33] examined self-disclosure of African-American students concerning campus life in a predominately "white" university with African-American (match) and Caucasian (mismatch) counselors. Participants with higher level of mistrust toward Caucasians provided a greater number of disclosures with African-American counselors. Another study found African-American respondents, who tested positive for cocaine use, to disclose more drug use, when interviewed by an African-American interviewer than by a Hispanic interviewer [32]. Other investigators demonstrated that ethnic matching may also produce a reverse effect [14]. In their study, respondents of Turkish and Moroccan origins in the Netherlands reported less alcohol use to ethnically similar interviewers in comparison with native Dutch interviewers. The authors explained this result by pointing to the Islam's prohibition of alcohol consumption in Turkish and Moroccan cultures.
The present study sought to investigate the EMs of Turkish and Moroccan Dutch patients in the context of the ethnic (mis)match with the interviewer. Past research among Turkish [17] and Moroccan [1] immigrants have pointed to a large degree of similarity in their EMs, pertaining to a wide range of supernatural, interpersonal, psychological and stress-related causal factors. Although the existing literature does not provide any clear hypotheses, given the principles of social tuning, one may expect a differential report of EMs in ethnic match and mismatch contexts. For instance, it seems plausible to assume that supernatural attributions (e.g., witchcraft and evil eye) may especially be susceptible to misreport to ethnically dissimilar interviewers, given their roots in cultural folk beliefs, which may appear primitive to outsiders. Hence, patients' tendency toward social desirable responding and the perceived quality of rapport with the interviewer may exert a greater influence on disclosure of these types of EMs. In addition, illness beliefs in general [34], and EMs in particular [38], are often fluid, ambiguous and uncertain cognitions that tend to be represented differently in response to varying interview contexts. In other words, due to the ambiguity of their EMs, patients may report different, even contrasting accounts at different moments or to different people. Taken this premise in light of the available evidence on social tuning, one may expect highly ambivalent individuals to manifest greater adjustment of their beliefs in response to interviewer characteristics, such as ethnicity.

A final factor of importance, which may affect the report of EMs in (mis)match contexts, pertains to patients' level of acculturation. Acculturation, roughly defined as cultural adaptations as a result of prolonged contact with the host society, presumably influences the types of EMs that patients hold [25], as well as the personal salience of an ethnically similar interviewer for individual minority patients. That is, an ethnic match situation may become less significant with increasing levels of acculturation.

In summary, the present study examined whether patients of Turkish and Moroccan origins reported different EMs in the match and in the mismatch situations. It was hypothesized that, regardless of patients' level of acculturation: (a) they would perceive supernatural EMs as more important in the match than in the mismatch condition; (b) the differences in reports of EMs in the match and mismatch contexts would be larger for patients with high tendency toward social desirability and (c) mediated by respondents' perceived quality of rapport with the interviewer; and finally (d) the (mis)match effect would be larger for participants who experience greater ambivalence toward their EMs. 


\section{Methods}

Design

Participants were randomly assigned (stratified according to gender and diagnosis) to one of two conditions: (1) match condition, in which participants were interviewed by an interviewer from the same ethnic background and (2) mismatch condition, in which a native Dutch interviewer conducted the interviews. Additionally, participants and interviewers were matched on gender. The research design and procedure were approved by our university ethical committee.

\section{Participants}

Participants were recruited among patients who were receiving treatment at two psychiatric outpatient centers in the city of Rotterdam. Patients were included, if they were 18 years or older, had at least one of their parents born in Turkey or Morocco, and had a DSM-IV diagnosis of major depression, dysthymia or any anxiety disorder at intake. Exclusion criteria were having a presumed psychotic disorder in active phase, or any severe cognitive disability, which would affect the quality of communication during the interview. Proficiency in the Dutch language was not an inclusion criterion.

\section{Interviewers}

Interviews were conducted by 17 interviewers, of whom 10 had native Dutch ethnicity (mean age $=24$ years, $\mathrm{SD}=1.15$ ), and 7 had Turkish or Moroccan (mean age $=25.28, \mathrm{SD}=5.52$ ) ethnicity. All interviewers in the match condition were graduate students in clinical psychology, whereas three interviewers in the mismatch condition were psychology students, and the remaining studied other disciplines (e.g., social science, economy). A 2-day extensive training was provided for all interviewers, in which the research procedure, and especially the interview protocol, were discussed.

\section{Instruments}

EMs were assessed, using the Explanatory Models Interview Catalogue (EMIC) [37]. This instrument consists of a collection of locally adapted semi-structured interview for eliciting EMs among specific cultural groups. The version, utilized in the present study, was partly based on a previously developed Turkish version [17], which was further adjusted for use among Turkish and Moroccan patients. The adjustment consisted of developing additional items, based on a review of the relevant literature and consultation with cultural experts. The interview consists of an open query into the perceived causes of patient's condition, and a checklist of 46 causal factors that were divided into 11 different categories of causes: ingestion of food or substances, medical, interpersonal, stress, loss and grief, migration-related factors, victimization (e.g., physical or sexual abuse), supernatural (e.g., djinns, evil eye), religious and mystical (e.g., divine punishment, fate), psychological (e.g., personality characteristics), and finally environmental causes (e.g., pollution). Each item was assigned a weighted numerical value according to EMIC standard scoring criteria, which has been extensively described elsewhere [37]. For each category of causes, a score was generated by calculating mean values for the individual item scores in that category.

Social desirability was measured with the short Dutch version [35, 39] of the Balanced Inventory of Desirable Responding (BIDR) [27]. This questionnaire consists of 24 items, and measures social desirability along two dimensions of impression management and self-deception. The reliability of the Dutch version is acceptable $(\alpha=0.79)$.

Quality of rapport was evaluated, using a short questionnaire, which was developed for the purpose of this study. Participants were asked to rate, on a five-point Likert scale $(1=$ completely disagree; 5 completely agree), whether they agreed with three statements pertaining to their level of comfort during the interview and the tendency to disclose information. In this sample, a Chronbach's alpha of 0.64 and a mean inter-item correlation of 0.37 were found for this scale.

Ambivalence toward EMs was assessed with a rating scale, which was integrated into the EMIC. On each item of the EMIC checklist, patients were asked to rate, on a fourpoint Likert scale, how likely they found the item to be a cause of their illness $(0=$ not at all; $4=$ very much $)$.

Acculturation was measured, using the Dutch, Turkish and Moroccan versions of the Lowlands Acculturation Scale (LAS) [26]. This measure was constructed and validated, based on research among the Turkish and Moroccan communities in the Netherlands. The LAS consists of 27 items that form the following subscales: social integration, traditions, norms and values, skills, and loss. Higher scores on these subscales reflect a greater orientation toward the culture of origin. Acceptable levels of reliability [19] and validity [26] have been reported for the entire scale.

Manipulation check was performed by one item added to the rapport questionnaire, which informed whether patient and interviewer had the same or a different ethnicity (yes/no).

Socio-demographic characteristics were assessed with a questionnaire focusing on age, gender, ethnicity, education and migration-related factors, such as participant's age at migration and the length of residence in the Netherlands. 
With the exception of the manipulation check and the socio-demographic characteristics, analyses were performed using the mean scores for each instrument. All measures, except the LAS, were translated into Turkish and Arabic, using the translation-back translation procedure.

\section{Procedure}

All interviews were conducted at the institutes where the participants were recruited. In order to isolate the interviewer effect, two different persons were involved in the data collection procedure. The first person welcomed the patient, and guided him or her to a room where the second person (the interviewer, introducing himself or herself as a student/research assistant) explained the procedure, acquired written informed consent, conducted the interview (EMIC), and subsequently administered the BIDR and LAS in random order. The socio-demographic characteristics were assessed at the end of the session. Finally, the interviewer left the room, and the first person returned to administer the rapport questionnaire. In order to make the ethnicity of the interviewer more salient in the match condition, the first person was always of native Dutch origin. For participants, who were not fluent in Dutch, a professional interpreter was available to facilitate the communications during the interview.

\section{Analyses}

\section{Preliminary analyses}

Analyses of potential non-response bias and randomization check were performed using Chi-square and $T$ tests. Further, a MANOVA was conducted to assess the effect of the interpreter's presence on the report of EMs in the mismatch condition. Finally, an additional MANOVA was performed to investigate possible differences in EMs of participants from Turkish and Moroccan origins.

\section{Main analyses}

In order to examine the effect of ethnic (mis)match on the report of EMs, data were initially analyzed by employing two different approaches: (1) separate multi-level regression analyses for each category of causes, which applied a correction for the nesting of multiple participants within individual interviewers (individual interviewer effect), and (2) a conventional MANCOVA, with acculturation scores as covariate, and scores on each of the 11 categories of causes as dependent variables. As both methods generated identical outcomes, only the results of the conventional analysis are presented in the next section. In case of significant differences, effect sizes were calculated using the Cohen's $d$. Interaction effects between social desirability and condition were tested by simultaneous multiple regression analyses, using condition, social desirability, their interaction term and acculturation as predictors, and each category of causes as the outcome variable. To assess whether the quality of rapport mediated the effect of ethnic (mis)match on EM reports, a series of regression analyses were conducted, using the following criteria, which were proposed by Baron and Kenny [3]: (1) the independent variable (condition) should predict the outcome variable (report of different types of EMs), (2) the independent variable should have an effect on the proposed mediator (perceived quality of rapport), and (3) the proposed mediator should predict the outcome variable, when controlling for the effect of the independent variable. Finally, interaction effects between ambivalence and condition were tested by simultaneous multiple regression analyses with condition, ambivalence, their interaction term and acculturation as predictors, and each category of causes as the outcome variable. Interaction effects were further analyzed by applying the Johnson-Neyman technique [18] in order to detect regions of significance on the moderating variable: that is, the values of the moderator (ambivalence) for which the scores in the outcome variable (EM categories) were significant for the match and mismatch groups.

\section{Results}

\section{Participants}

A total of 121 patients were approached for participation, of whom 66 refused $(54.5 \%)$. Reasons provided for refusal were fatigue $(34.8 \%)$ and no time or opportunity (51.5\%). Six patients $(9.1 \%)$ did not disclose a reason, and in three cases $(4.5 \%)$ the reason for refusal was not recorded. No significant differences were found between participants and refusers on age, gender, ethnicity and diagnosis.

Three patients in the mismatch condition reported to have been interviewed by an ethnically similar interviewer on the manipulation check. Their data were excluded from further analysis, so that the final sample consisted of 52 patients. Another participant was excluded from the analysis of social desirability scores, due to excessive number of missing values on the BIDR.

Among the participants in the mismatch condition, 12 (48\%) were assisted by an interpreter during the interviews. However, the overall effect of the interpreter's presence on the EM scores of respondents in the mismatch condition was not significant. In addition, analyses found no 
Table 1 Sample characteristics for the match and mismatch conditions $(N=52)$

\begin{tabular}{|c|c|c|c|c|}
\hline & \multicolumn{4}{|c|}{ Condition } \\
\hline & \multicolumn{2}{|c|}{ Match $(n=27)$} & \multicolumn{2}{|c|}{ Mismatch $(n=25)$} \\
\hline & $n$ & $\%$ & $n$ & $\%$ \\
\hline \multicolumn{5}{|l|}{ Gender } \\
\hline Male & 10 & 37.0 & 11 & 44.0 \\
\hline Female & 17 & 63.0 & 14 & 56.0 \\
\hline \multicolumn{5}{|l|}{ Ethnicity } \\
\hline Turkish & 15 & 55.6 & 14 & 56.0 \\
\hline \multirow[t]{2}{*}{ Moroccan } & 12 & 44.4 & 11 & 44.0 \\
\hline & $M$ & SD & $M$ & SD \\
\hline Age & 41.9 & 7.8 & 45.6 & 7.9 \\
\hline Years of education & 6.5 & 3.5 & 6.8 & 6.3 \\
\hline Age at migration & 17.3 & 8.1 & 19.9 & 8.5 \\
\hline Years in the Netherlands & 24 & 8.6 & 25.1 & 7.7 \\
\hline Social desirability & 3.27 & 0.4 & 3.4 & 0.4 \\
\hline Acculturation & $4.3^{*}$ & 0.7 & 3.9 & 0.7 \\
\hline
\end{tabular}

$* P=0.05$

difference in the EMs of participants of Turkish and Moroccan origins.

Sample characteristics are shown in Table 1. All participants were (first-generation) migrants, with both their parents born and raised in Turkey or Morocco. No significant differences were observed between participants in both conditions, except for the level of acculturation. Patients in the match condition appeared to be less acculturated than those in the mismatch condition, $t(50)=2.03$, $P=0.05$.

\section{Effect of ethnic (mis)match on the disclosure of EMs}

An overall effect of ethnic (mis)match was found on the report of EMs, $F(11,39)=2.83, P<0.01$. Contrary to the first hypothesis, participants in the match condition did not perceive supernatural causes as more important than those in the mismatch condition. However, a number of significant differences were found on other categories of EMs, regardless of participants' level of acculturation (Table 2). Patients in the match condition scored higher on the interpersonal $(d=0.70)$, victimization $(d=0.90)$ and religious/mystical causes $(d=0.70)$. In contrast, participants in the mismatch condition had higher scores on medical causes $(d=0.56)$.

No interaction effects were found between social desirability and condition for any category of EMs. However, social desirability had an independent effect on the report of interpersonal $(\beta=-0.31, P=0.02)$ and
Table 2 Differences between the match and mismatch conditions on the EM category scores $(N=52)$

\begin{tabular}{|c|c|c|c|c|c|c|}
\hline & \multicolumn{4}{|c|}{ Condition } & \multirow[b]{3}{*}{$F(1,49)$} & \multirow[b]{3}{*}{$P$} \\
\hline & \multicolumn{2}{|c|}{$\begin{array}{l}\text { Match } \\
(n=27)\end{array}$} & \multicolumn{2}{|c|}{$\begin{array}{l}\text { Mismatch } \\
(n=25)\end{array}$} & & \\
\hline & $M$ & $S D$ & $M$ & $S D$ & & \\
\hline Ingestion & 0.54 & 0.83 & 0.41 & 0.68 & 0.48 & 0.49 \\
\hline Medical & 0.74 & 0.76 & 1.12 & 0.59 & 4.71 & 0.03 \\
\hline Interpersonal & 2.12 & 1.65 & 1.14 & 1.08 & 6.10 & 0.02 \\
\hline Stress & 1.40 & 1.07 & 1.55 & 0.76 & 0.56 & 0.46 \\
\hline Migration & 1.42 & 1.23 & 0.94 & 1.24 & 0.66 & 0.42 \\
\hline Loss & 1.24 & 1.02 & 1.04 & 1.07 & 0.71 & 0.40 \\
\hline Victimization & 0.92 & 0.97 & 0.24 & 0.45 & 12.00 & $<0.01$ \\
\hline Supernatural & 0.80 & 1.01 & 0.80 & 0.99 & 0.33 & 0.57 \\
\hline Religious/mystical & 1.91 & 0.86 & 1.33 & 0.79 & 4.96 & 0.03 \\
\hline Psychological & 1.49 & 0.78 & 1.31 & 0.81 & 1.23 & 0.27 \\
\hline Environmental & 0.81 & 0.86 & 0.71 & 0.77 & 0.37 & 0.54 \\
\hline
\end{tabular}

victimization causes $(\beta=-0.25, P=0.05)$, with high levels of social desirability predicting less disclosure.

In order to examine whether the effect of ethnic (mis)match on the report of medical, interpersonal, religious/ mystical and victimization EMs was mediated by the perceived rapport, a series of regression analyses were conducted, corresponding to criteria 2 and 3 for mediation effects. The first analysis revealed a significant relationship between ethnic (mis)match and the quality of rapport, meeting criterion 2 ; participants in the match condition perceived the rapport with the interviewer more positively than those in the mismatch condition, $\beta=-0.43$, $P=0.05$. However, when controlling for the effect of ethnic (mis)match (criterion 3), the quality of rapport did not predict the report of any types of EMs. Hence, a mediating effect of rapport could not be established for the (mis)match effect on any category of causes.

Effect of ethnic (mis)match and ambivalence on report of EMs

In line with the hypothesis, the examination of interaction effects between ethnic (mis)match and level of ambivalence toward each category of causes revealed two significant outcomes. First, a significant interaction effect was observed with respect to the scores on medical causes (condition $\times$ ambivalence: $\beta=0.25, P=0.03$ ). Applying the Johnson-Neyman technique, an upper region of significance was found for all values of ambivalence above 1.58 ( $0.58 \mathrm{SD}$ above the mean). This finding indicates that as ambivalence scores exceeded 1.58 , participants in the mismatch condition scored significantly higher on medical 
causes than those in the match condition $(\beta=0.23$, $P=0.05)$.

Second, a reverse interaction effect emerged on scores on religious/mystical causes (condition $\times$ ambivalence: $\beta=-0.28, P=0.03$ ). An upper region of significance was observed for all values of ambivalence above 2.33 (0.08 SD above the mean), indicating that with ambivalence scores exceeding 2.33, participants in the match condition scored significantly higher on religious/mystical causes than their counterparts in the mismatch condition $(\beta=-0.27, P=0.05)$.

\section{Discussion}

The present study examined the effect of ethnic (mis)match between interviewer and respondent on the report of EMs of mental illness. Contrary to the hypothesis, however, participants in the match condition did not perceive supernatural causes as more important than those in the mismatch condition. Patients scored higher on interpersonal, victimization and religious/mystical causes, when interviewed by an ethnically similar interviewer, and scored higher on medical causes, when interviewed by an ethnically dissimilar interviewer. The data provided no evidence for the moderating role of social desirability; high levels of social desirability appeared to be related to less disclosure of victimization and interpersonal causes, regardless of the ethnicity of the interviewer. Contrary to the hypothesis, the perceived quality of rapport did not seem to mediate the (mis)match effect. Finally, as expected, patients who experienced greater ambivalence toward their EMs scored higher on religious/mystical causes in the match, and higher on medical causes in the mismatch condition.

The absence of a (mis)match effect in the report of supernatural EMs was unexpected and may have arisen from a number of factors. First, the interviewer's mere probing of specific checklist items of the supernatural category in the mismatch condition, sometimes in the respondent's own native language to enhance communication, may have demonstrated a certain level of familiarity or recognition on the part of the interviewer, thus facilitating the report of these types of EMs. Alternatively, one can argue that participants in the match condition were equally reserved about disclosing supernatural EMs in a medical setting, when facing a younger person who may not endorse traditional folk beliefs.

However, the data provided evidence of (mis)match effect regarding a number of other categories of causes. It is important to note that different categories of EMs pertain to various aspects of individual's life and functioning, some of which may be more private or socially/culturally sensitive than others. It seems, therefore, reasonable to assume that reports of different types of EMs in (mis)match situations may not share the same underlying mechanism. The differential reports of medical and religious/mystical EMs in match and mismatch situations may have resulted, more than from any other category of causes, from social tuning. More specifically, given the higher endorsement of religious/mystical causes in the match situation, one may assume that patients felt more pressured to acknowledge religious causes before a perceived fellow Muslim. This result has significant implications for the interpretation of previous research findings, which point to a relatively high prevalence of religious attributions among Turkish [25] and other non-Western respondents $[6,21]$. As these studies have all employed ethnically similar interviewers, high reports of religious EMs may not necessarily reflect genuine beliefs of the respondents, but merely be an artifact of the data collection method (i.e., ethnic matching). A similar process may underlie the participants' higher reports of medical EMs in the mismatch situation. Patients may have tuned their accounts toward the perceived views of a native Dutch interviewer in a medical setting. Interestingly, with respect to both medical and religious/mystical causes, high ambivalence toward one's own beliefs seemed to magnify social tuning. This finding indicates that the adjustment of health beliefs in the interview context [34] may partly occur due to the uncertain nature of these cognitions, which makes their report more susceptible to the interviewer characteristics.

In contrast, interpersonal and victimization causes both pertain to private aspects of patients' lives, and are unlikely to result from social tuning tendencies. One may assume that the differential report of these causes in the (mis)match situations would be related to the perceived rapport during the interview. This appeared, however, not to be the case. The higher disclosure rate of victimization causes contradicts previous research findings. Dailey and Claus [11] found no effect of ethnic matching on the disclosure of physical and sexual abuse among Caucasian and AfricanAmerican respondents. Besides cultural and demographic differences in the study samples, the combination of ethnic and gender matching in the present study may have been crucial for the match effect to occur.

Overall, the study failed to clarify the factors, contributing to the (mis)match effects. Social desirability appeared to impact patients' accounts only with regard to interpersonal and victimization causes in both match and mismatch situations. Perhaps, these types of EMs include such sensitive information that social desirability can affect their disclosure by itself, independent of the ethnicity of the interviewer. Furthermore, given the (mis)match effect was not moderated by social desirability, one may hypothesize that certain belief adjustments in (mis)match situations 
may have not been necessarily deliberate, but in fact have occurred outside the patients' conscious attempts to manipulate their utterances. Indeed, social psychological literature seems to support this assertion, indicating that belief and attitude adjustments in interpersonal interactions may be unintended [22], or occasionally even counter a socially desirable self-presentation [31]. Nonetheless, even in cases in which adjustments are not deliberate, social desirability can be assumed to affect the individual's statements [24], albeit perhaps not as profoundly as the case with conscious manipulations.

The study found no relation between the perceived rapport and patients' statements in (mis)match situations. Good rapport between interviewer and respondents has been previously thought to foster disclosures [13] by countering social desirability tendencies, thus making patients' expressions less threatening [9]. A number of additional factors may explain this discrepancy. First, the quality of rapport was measured after the completion of the interview, whereas EMs were assessed at the beginning of the encounter, when a rapport may not have been fully established yet. It is, therefore, not surprising that reports of EMs at one moment do not seem to be associated with the evaluation of rapport at a later moment. A second factor pertains to the theoretically complex relationship between rapport and disclosure. Similar to the present findings, a number of studies have found rapport not to predict response accuracy [4], or to mediate the relationship between interviewer characteristics and patient's disclosure rate [28]. Such findings have led a number of authors (e.g., [13]) to propose a curvilinear relationship between rapport and disclosure, in which very low and very high rapport may both inhibit disclosure. Indeed, the lack of evidence for the mediating role of rapport in the present study may be due to respondents' reluctance to disclose information, which would have undermined a positive rapport with the interviewer, insofar as it has already been established.

This study suffers from a number of limitations. First, the sample size was not large. It is possible that social desirability and rapport would be more strongly linked to disclosure, had a larger sample size been acquired. Second, participants in this study were all immigrants. Data may not be generalizable to the second-generation of Turkish and Moroccan Dutch individuals, among whom the ethnic (mis)match effect may be less pronounced.

\section{Conclusion}

This study provided evidence for the effect of ethnic (mis)match between interviewer and respondent on the report of EMs among psychiatric patients of Turkish and Moroccan origins. Although the exact mechanism underlying this effect remains largely unknown, the study has a number of significant implications for clinical research and practice. First, researchers should be aware of (mis)match effects in the study of EMs, and possibly other similar constructs of interest. Ethnic matching of interviewers and respondents may elicit more genuine accounts on some subjects (e.g., victimization causes), while resulting in social tuning on others (e.g., religious/mystical causes). Particularly, in cross-cultural comparisons, such tunings of beliefs and utterances may generate false assumptions of cultural differences. Therefore, possibility of (mis)match effects should, ideally, be included in decisions regarding the research procedure and interviewer selection. Second, mismatch effects are not merely a source of non-random measurement error, but also reflect important dynamics in intercultural clinical encounters. Attempts should be made to enhance disclosure in the starting phase of these encounters in order to facilitate a more valid assessment of the patient's EMs and history. Further studies into the mechanisms of (mis)match effects will be essential for this purpose.

Acknowledgments This work was supported by a grant from the Netherlands Organization for Scientific Research. The authors would like to thank Fatima Ahyad, Ajoes Jankie and Carien Pille at Bavo RNO Groep, and Michael van den Boogaard, Angelina Franken and Dr. Cor Hoffer at GGZ Groep Europoort in Rotterdam. Gratitude is expressed to Drs. Mitchell Weiss and Daniel Maeusezahl and their colleagues for grating permission to use and adjust the Explanatory Models Interview Catalogue.

Open Access This article is distributed under the terms of the Creative Commons Attribution Noncommercial License which permits any noncommercial use, distribution, and reproduction in any medium, provided the original author(s) and source are credited.

\section{References}

1. Al-Krenawi A (2001) Ethnic and gender differences in mental health utilization: the case of Muslim Jordanian and Moroccan Jewish Israeli out-patient psychiatric patients. Int J Soc Psychiatry $47: 42-54$

2. Anderson BA, Silver BD, Abramson PR (1988) The effects of the race of the interviewer on race-related attitudes of black respondents in SRC/CPS national election studies. Public Opin Q 52:289_ 324

3. Baron RM, Kenny DA (1986) The moderator-mediator variable distinction in social psychological research: conceptual, strategic, and statistical considerations. J Pers Soc Psychol 51:11731182

4. Belli RF, Lepkowski JM, Kabeto MU (2001) The respective roles of cognitive processing difficulty and conversational rapport on the accuracy of retrospective reports of doctor's office visits. In: Cynamon ML, Kulka RA (eds) Seventh Conference on Health Survey Research Methods. Government Printing Office, Hyattsville, MD

5. Bhui K, Bhugra D (2002) Explanatory models for mental distress: implications for clinical practice and research. Br J Psychiatry 181:6-7 
6. Bhui K, Bhugra D, Goldberg D (2002) Causal explanations of distress and general practitioners' assessments of common mental disorder among Punjabi and English attendees. Soc Psychiatry Psychiatr Epidemiol 37:38-45

7. Blair IV (2002) The malleability of automatic stereotypes and prejudice. Pers Soc Psychol Rev 6:242-261

8. Callan A, Littlewood R (1998) Patient satisfaction: ethnic origin or explanatory model? Int J Soc Psychiatry 44:1-11

9. Cannell CF, Miller PV, Oksenberg L (1981) Research on interviewing techniques. Sociol Methodol 12:389-437

10. Chesla CA (1989) Parents' illness models of schizophrenia. Arch Psychiatr Nurs 3:218-225

11. Dailey RM, Claus RE (2001) The relationship between interviewer characteristics and physical and sexual abuse disclosures among substance users: a multilevel analysis. J Drug Issues 31:867-888

12. Davis DW (1997) The direction of race of interviewer effects among African-Americans: donning the black mask. Am J Pol Sci 41:309-322

13. Dijkstra WIL (1987) Interviewing style and respondent behavior: an experimental study of the survey interview. Sociol Methods Res 16:309-334

14. Dotinga A, Van Den Eijnden RJJM, Bosveld W, Garretsen HFL (2005) The effect of data collection mode and ethnicity of interviewer on response rates and self-reported alcohol use among Turks and Moroccans in the Netherlands. Alcohol Alcohol 40:242-248

15. Finkel SE, Guterbock TM, Borg MJ (1991) Race-of-interviewer effects in a preelection poll: Virginia 1989. Public Opin Q 55:313-330

16. Foulks EF, Persons JB, Merkel RL (1986) The effect of patients' beliefs about their illnesses on compliance in psychotherapy. Am J Psychiatry 143:340-344

17. Gilgen D, Maeusezahl D, Salis Gross C, Battegay E, Flubacher P, Tanner M, Weiss MG, Hatz C (2005) Impact of migration on illness experience and help-seeking strategies of patients from Turkey and Bosnia in primary health care in Basel. Health Place $11: 261-273$

18. Huitema BE (1980) The analysis of covariance and alternatives. Wiley, New York

19. Kamperman AM, Komproe IH, De Jong JTVM (2003) De relaie tussen culturele aanpassing en psychische gezondheid bij eerste generatie Turkse, Marokkaanse en Surinaamse migranten [The relation between cultural adaptation and psychological health among first-generation Turkish, Moroccan, and Surinamese migrants]. Gedrag en Gezondheid 31:163-174

20. Kleinman A (1980) Patients and healers in the context of culture. University of California Press, Berkley

21. Lloyd KR, Jacob KS, Patel V, St LL, Bhugra D, Mann AH (1998) The development of the Short Explanatory Model Interview (SEMI) and its use among primary-care attenders with common mental disorders. Psychol Med 28:1231-1237
22. Lowery BS, Hardin CD, Sinclair S (2001) Social influence effects on automatic racial prejudice. J Pers Soc Psychol 81:842-855

23. McCabe R, Priebe S (2004) Explanatory models of illness in schizophrenia: comparison of four ethnic groups. Br J Psychiatry 185:25-30

24. McCann CD, Hancock RD (1983) Self-monitoring in communicative interactions: social cognitive consequences of goal-directed message modification. J Exp Soc Psychol 19:109-121

25. Minas H, Klimidis S, Tuncer C (2007) Illness causal beliefs in Turkish immigrants. BMC Psychiatry 7:34

26. Mooren TTM, Knipscheer J, Kamperman AM, kleber R, Komproe IH (2001) The Lowlands Acculturation Scale: validity of an adaptation measure among migrants in the Netherlands. In: Mooren TTM (ed) The impact of war studies on the psychological consequences of war and migration. Eburon, Delft, pp 49-68

27. Paulhus DL (1984) Two-component models of socially desirable responding. Journal of Personality and Social Psychology pp. 598-609

28. Pollner MPD (1998) The effects of interviewer gender in mental health interviews. J Nerv Ment Dis 186:369-373

29. Rose LE (1983) Understanding mental illness: the experience of families of psychiatric patients. J Adv Nurs 8:507-511

30. Saravanan B, Jacob KS, Johnson S, Prince M, Bhugra D, David AS (2007) Belief models in first episode schizophrenia in South India. Soc Psychiatry Psychiatr Epidemiol 42:446-451

31. Sinclair S, Huntsinger J, Skorinko J, Hardin CD (2005) Social tuning of the self: consequences for the self-evaluations of stereotype targets. J Pers Soc Psychol 89:160-175

32. Tassiopoulos K, Bernstein J, Heeren T, Levenson S, Hingson R, Bernstein E (2006) Predictors of disclosure of continued cocaine use. Addict Behav 31:80-89

33. Thompson CE (1994) Counselor content orientation, counselor race, and black women. J Couns Psychol 41:155-161

34. Van der Geest S (1991) Marketplace conversations in Cameroon: how and why popular medical knowledge comes into being. Cult Med Psychiatry 15:69-90

35. Vorst (2002) Nederlandse Aanpassing van de BIDR [Dutch Adaptation of the BIDR]. Unpublished report, Department of Psychology, University of Amsterdam, Amsterdam

36. Weeks MF, Moore RP (1981) Ethnicity-of-interviewer effects on ethnic respondents. Public Opin Q 45:245-249

37. Weiss M (1997) Explanatory Model Interview Catalogue (EMIC): framework for comparative study of illness. Transcult Psychiatry 34:235-263

38. Williams B, Healy D (2001) Perceptions of illness causation among new referrals to a community mental health team: "explanatory model" or "exploratory map"? Soc Sci Med 53:465-476

39. Yildirim V (2005) Struikelblokken in de Klachtenpresentaties van Allochtone Patiënten [Obstacles in the Symptom Presentations of Ethnic Minority Patients]. Unpublished master's thesis, University of Amsterdam, Amsterdam 